\title{
An Extensible Healthcare Context Engine Based on Smart Phones
}

\author{
Zheng Guangbin, Muhammad Arshad Awan, and Shin-Dug Kim
}

\begin{abstract}
Population aging problem has become a severe challenge worldwide and will become even severer in the near future. Meanwhile the technology of smart phones and various sensors is developing by leaps and bounds in the last few years. In order to help people who have difficulty in living independently, especially the senior citizens and facilitate efficient use of these sensors, it is worthwhile to explore the possibility of making use of smart phones to manage these sensors and provide valuable information. In this paper, a context engine run on the Android platform is introduced. The context engine targets healthcare usage, collecting healthcare related information from three kinds of sensors: ambient sensors, wearable sensors, and embedded sensors, with the attention of providing real time reminders, sharing information to other applications and recording the user's daily life. Experiment result shows that average accuracy of reminders can reach $82 \%$ in user's daily life within the context engine. The results are expected to be improved with more sensors and more accurate sensors involved in the system. Compared to other similar work, this context engine focuses on the mobile platform that can cover most of user's time in daily time while maintaining portability.
\end{abstract}

Index Terms - Context aware application, context engine, context information, information abstraction.

\section{INTRODUCTION}

The world population is aging very quickly, and demographics have changed much in the last 30 years. This population aging problem is most significant in the most highly developed countries. It is expected that within twenty years Asia and Europe will have many countries facing situations, where the largest population cohort will be those over 65 and have an average age approaching 50 years. It's very challenging to meet these senior citizens' health care needs. It seems that it will become necessary for senior citizens to take more actions to be able to live independently.

In the last decade, smart phones have become very common and convenient devices with high usability. Even senior citizens have very few obstacles in using them. Meanwhile, more and more sensors, especially sensors related to the ambient environment and physiological activity, are available. What is more, the processing capacity for the current smart phones is no less than PC's. They can do most of the work that we previously did on PC, but smart phones maintain portability. All of these including, popularity, processing capacity, high usability and multiple network

Manuscript received June 5, 2013; revised September June 25, 2013.

Zheng Guangbin, Muhammad Arshad Awan, and Shin-Dug Kim are with the Department of Computer Science, Yonsei University, Seoul, Korea (e-mail: zhenggb@naver.com, m99arshad@hotmail.com, sdkim@yonsei.ac.kr). compatibility of smart phones make them perfect devices to assist senior citizens in their daily lives.

To use the sensor technology and help senior citizens to live more independently and conveniently, a context engine run on smart phones has been made by the author. The context engine tries to collect as much information from all possible sensors related to healthcare and gives other context aware applications running on the same platform the ability to adapt to changing context information. Context aware applications are described as intelligent applications that can monitor the user's context and, in case of changes in this context, consequently adapt their behavior in order to satisfy the user's current needs or anticipate the user's intentions [1].

The context engine collects related data at a necessary frequency, abstracts the low level data to high level, and combines some simple context information to composite one. This data can then be used by the context engine itself by giving real time reminders or alarms, or can be used by third-party applications using the context engine and focusing on a special group of user with special need. This data can also be used to keep real time and an evidence-based record of the user's daily life. This would be of great value for the user's future medical treatment. Experiment result shows that average accuracy of reminders can reach $82 \%$ in user's daily life within the context engine. The results are expected to be improved with more sensors and more accurate sensors involved in the system.

\section{RELATED WORK}

There has been much research done on the application of sensors in the health care field since the emergence Wireless Sensor Network (WSN). G. Virone proposed an assisted living oriented information system based on a residential wireless sensor network [2]. In this work the author dealt with a system called Alarm Net which targets remote health monitoring for assisted-living residents. The system confirmed the potential of usage for WSNs on the medical care field, however it lacks of portability. In the paper of "a smart wireless sensor network for AAL"[3] the author implemented a novel system to assist weak people in exploring indoor environment. The system extracts suitable information through the multi-sensor based on a WSN and smart paradigm. A validation concept for algorithms and sensors that detect emergencies and monitor long term health development was proposed in [4]. Two validation methods for short- and long-term health monitoring using ambient sensor technology was defined in this paper. A context model was brought up in [5] in order to manage the increasing impact of chronic conditions. In this work, an ontology-based 
context model and a related context management middleware was described. The middleware provides an application framework assisting and monitoring patients at home

The concept of context aware applications has become very popular with the usage of sensor networks, and health care is one of the important issues in this subject. In [6] a multi-level model to build context-aware self-adaptive entities was proposed. In this paper, mandatory functionalities for adaptive entities were defined in basic level while the upper-level focus on coordination of composite entities and primitive entities.

In these researches list above, it is very obvious that the server holds an important place in the running of the system, although the authors did not emphasize this. The server has to manage all the sensors and process the information collected. The server is usually placed inside of the user's home. By doing this, the system could lose its mobility. In people's real life, much of the user's time is spent outside of his/her home. If the system is fixed in home, this part time is out of monitoring. In recent years, a few studies trying to make use of the smart phone to gain the mobility while providing full time service have been done. ContextDroid [7] was introduced with the attention of provide the application developers a framework with the services required to build context aware applications. In [8], a generic context acquisition engine was proposed. It is capable of context capturing, composition and broadcasting. In the above two work, context engine on the mobile platform is universal and can be used by other applications, thus it is less possible for it to provide better service by focusing on a special field. In this paper a healthcare oriented context engine is introduced. By focusing only on the healthcare, many user customized services can be provided.

\section{ARChitecture of THE CONTEXT ENGINE}

\section{A. The Three Types of Sensors}

The authors, wishing to make the data collected by the context engine reliable and ubiquitous in most healthcare scenarios, believe that application to as many types of sensors as possible should be investigated. Therefore, the authors have covered a wide range of sensors in the design of context engines. Here all sensors can be classified into three types: ambient sensors, wearable sensors, and embedded sensors.

Ambient sensors refer to sensors that are commonly used in the domestic environment. These sensors can collect the environmental information in the user's activity range. Sensors like temperature sensors, force sensors, light sensors and proximity sensors fall into this category. With the information collected by these sensors, context information like "What is the temperature now?", "Did the user enter into the bathroom?", and "Is the user sleeping in his /her bed?" can be derived.

Wearable sensors include sensors that can be embedded in the user's clothes or accessories and will not cause much inconvenience to the user when worn, such as heart rate sensors and blood pressure sensors embedded in user's clothes. The wearable sensors can provide most of the vital signs context information with acceptable accuracy.
Moreover, growing and recent research shows that by using a set of gravity sensors and accelerators worn by the user, the user's current activity can be precisely analyzed[11]. Knowing the user's current activity is very helpful and important to the context engine.

Embedded sensors as investigated here especially denote those sensors that are embedded within the user's smart phone. They are the most convenient type of sensors that can be directly used by the context engine. In recent years, multifarious sensors are being embedded into smart phones, aside from the commonly used GPS sensors, magnetic sensors, accelerometers. Many other sensors such as barometric sensors and UV sensors are beginning to appear in smart phones as of late.

The three types of sensors mentioned above use their own communication protocols to transfer data which may not be supported by the smart phone. For instance, some sensors use Zigbee as their communication protocol. This protocol is not supported by most smart phones. In such a case it would be necessary to implement a gateway from Zigbee to Bluetooth [9]. In this paper, it is assumed that all sensors can communicate with the smart phone without having to implement a gateway. The implementation of gateways is beyond the scope of this paper.

Apart from the three types of sensors above, it is possible to obtain context information from a few other data source. First, before usage of the context engine, the user must provide some basic information including age, gender, weight, physical condition, and preferences, etc. Second, since the smart phone can access to the internet, it is possible to collect some useful information from the internet. For instance, if the user does not have an outdoor ambient temperature sensor, it is possible to use the public temperature data from local meteorological departments through internet. Although it may be not as accurate as a specially installed temperature sensor, it still has acceptable reference value. Information source material of this kind can be called a virtual sensor [10].

\section{B. Architecture}

The Fig. 1 above gives a general description of how the context engine works with the other part of the system. As you can see, there is a smart phone depicted in the center which manages the sensors, collects raw data from the three types of sensors, processes the raw data, and abstracts the raw data to a higher level. By using these two data sets, it gives real time reminders to the user and can also provide APIs to the application using the context engine. User data is also sent into the cloud. There on the cloud side it becomes possible to do more advanced user's behavior analysis.

Fig. 2 in the following explains the components of context engine and how these components works together. The Sensor Manager manages all sensors, registers new sensors, disconnects unnecessary sensors, and adjusts data collecting frequency according to data request of other application. The Data Analyzer deals with requests from other applications, collects raw data from necessary sensors in an appropriate frequency, eliminates outliers, and abstracts the raw data to higher level data. With some predefined rules and the basic context information, it is possible to derive composite context 
information. For instance, with basic context information from the temperature sensors built onto both indoors and outdoors, it is possible to derive context information on the difference between the indoor and outdoor temperature. This difference would understandably be very important to users going out.

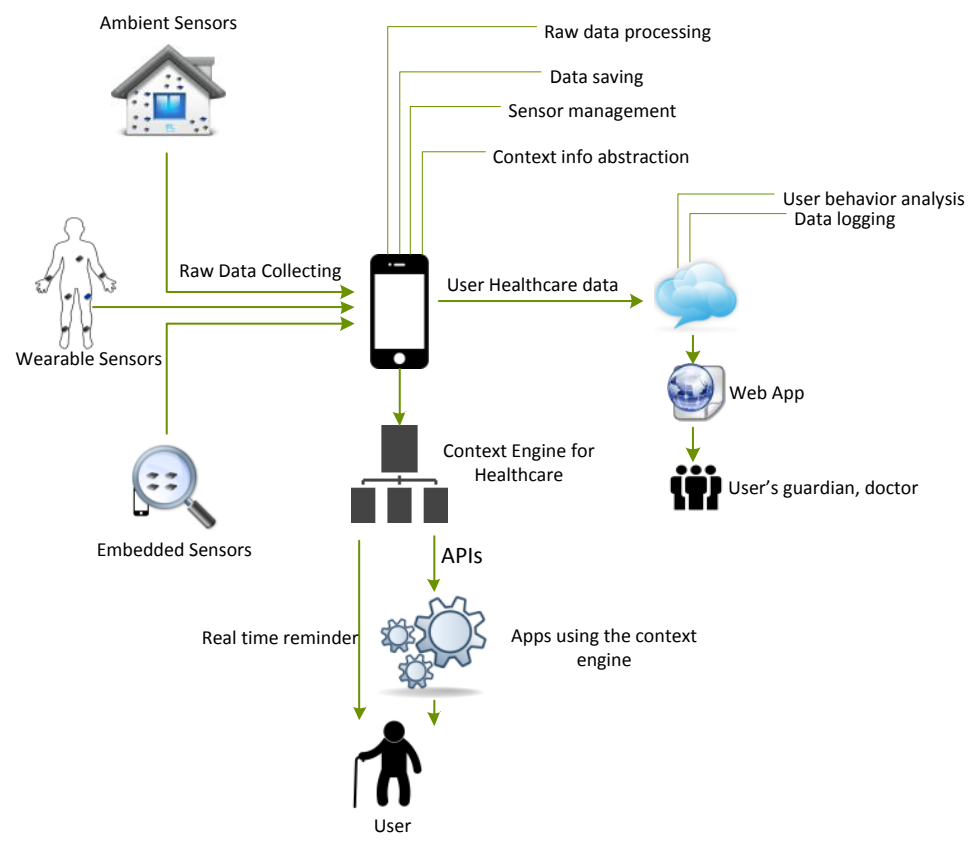

Fig. 1. The architecture of the context engine.

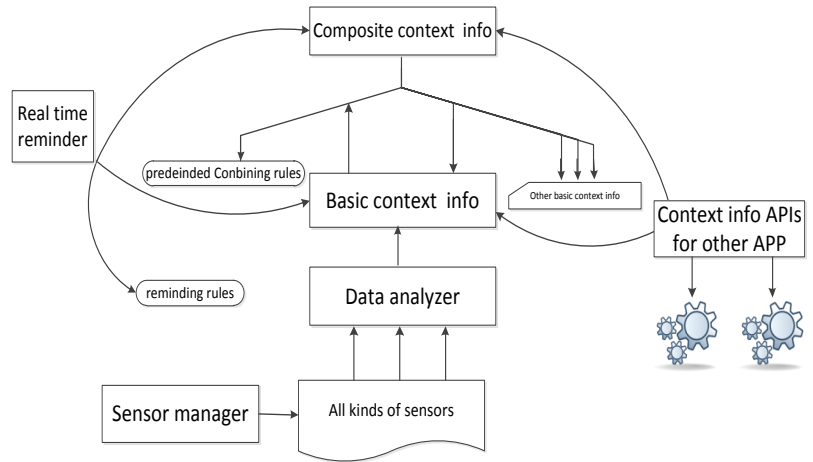

Fig. 2. Components for the context engine.

\section{PROCESSING OF THE CONTEXT INFORMATION}

\section{A. Information Entity}

In the context engine, all context information is defined as information entity. Information entity is not necessarily connected to a specific sensor hardware. It only indicates a type of sensible data source. For instance, some important data such as the heart rate may need two sensors to sense the same information for redundancy. In this case, the information entity is heart rate. It relates to two sensors. In this example, if the two hardware sensors are removed from the system, the information entity that indicates the heart rate still exists only its status would indicate inactivated. The Sensor Manager component manages the relationship between the information entity and the hardware sensor. By designing the architecture this way, the upper level of the context engine does not need to communicate with the underlying level of sensor hardware. Each information entity was defined in the flowing way. <entity id, entity name, type, range of influence, maximum collecting frequency, status $>$ Here the type reflects one of the three types of sensors mentioned above. Range of influence is defined as the effective coverage of the information entity. The following are two examples of the information entity:

$<$ tem010, bedroom temperature, ambient, bedroom, every 5 seconds, activated>

<vs021, blood glucose, wearable, user002, every 1 minute, inactivated>

In the design, a very important information entity is the activity entity. This entity indicates the current activity that the user is performing. This activity entity is mostly derived from the data value of wearable accelerometers. Many daily activities can be precisely analyzed such as walking, sitting, reclining, jogging, falling and walking upwards (e.g. ascending the stairs). Another team in the same laboratory with the author is focusing on this activity recognition. In the context engine, the activity entity is defined:

$<$ hum001, human activity, wearable, user001, every 3 seconds, activated $>$

\section{B. Information Abstraction}

For most sensors, the primitive value of the data is nothing but fluctuations in voltage value. These values can be transformed to the requested value by the driver of the sensor or programs run on the data request side. But that is far from enough. For instance, one can always just read the indoor temperature sensor to get the temperature value in Celsius. It is not done, as most applications are not interested in just the detailed temperature value. A strict value does not lend any further actionable information. What is really needed is data in a higher level, such as cold, hot, comfortable.

In the system, the abstraction is performed in the following way. For the information entity that needs to be abstracted, the abstraction rules must be predefined. By calculating the raw data using the predefined rules, this outputs another set of higher level data. In the temperature case the rules are 
predefined as follows:

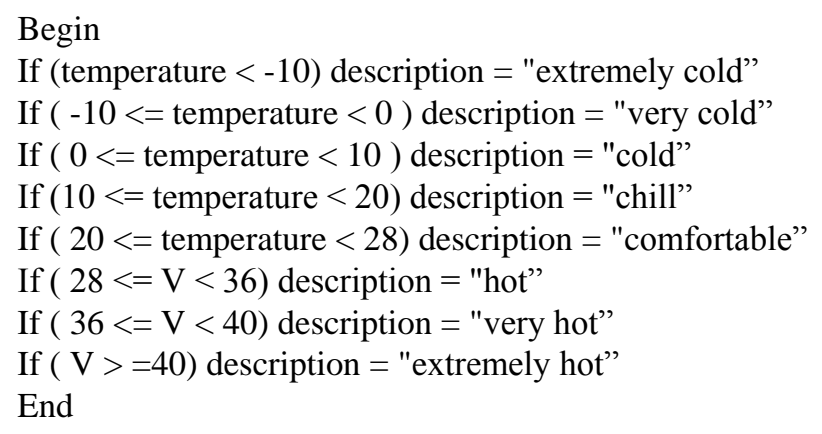

The predefined rules above may apply for most people, but each person has a slightly different perception of temperature. It is therefore important to only predefine the rules in the context engine while giving a right to edit the rules to the user according to their own tastes.

By applying the rules above, a higher level of the temperature data can be easily gotten. In some cases, abstracting the information to this level is still not enough. For example, sometimes it will be very convenient to calculate with context information from different sensors. This requires abstracting context information to an even higher level. Take the temperature example again. Further abstracting of the information entity can be done in the following way:

Define: Unbearable! $=2$, Endurable $!=1$, Comfortable $=0$ Begin:

If temperature is in \{extremely cold, extremely hot, very cold, very hot $\}$ temperature $=$ Unbearable!

If temperature is in $\{$ cold, chill, hot $\}$ temperature $=$ Endurable!

If temperature is in $\{$ comfortable $\}$ temperature $=$ Comfortable!

End

\section{Composite Information Entity}

Information entity such as ultraviolet intensity, value collected directly from hardware sensors, is defined as basic information entity. For information entity such as environmental amenity, value is a compilation of several information entities, such as temperature, humidity, noise, ultraviolet intensity, etc. These are not collected directly from a hardware sensor. Information entity of this type is called composite information entity in the context engine.

Composite information entity is dealt with in the following way: listing the basic information entities for the composite information entity, abstracting basic information entity to the highest level and obtaining the composite information entity by calculating the value of abstracted basic information entities. For the environmental amenity basic information entities are defined in the following: $<$ com003, environmental amenity, EF $>\mid<$ tem026, temperature, temp >, <hum004, Humidity, humi >, <noise006, Noise, noi>, <ult001, Ultraviolet, ult>>

In the process of information abstraction one can eventually abstract the basic information to the highest level of a set of about three enumerable values with descriptions. For instance, for the ultraviolet intensity, if the result set is $\{$ Harmful!=2, Careful! $=1$, Ignorable $=0\}$, for temperature, the result set is $\{$ Unbearable!=2, Endurable!=1, Comfortable $=0\}$. Therefore, if the four information entities are: temp $=0$, humi $=0$, noi $=1$, ult $=2$. The environmental amenity is found by calculating Max \{temp, humi , noi , ult $\}$

The answer is obviously (2), which indicates the environmental amenity now is suboptimal for health. Here, composite information entity is obtained by a simple calculation of the three high levels of data.

\section{APPLICATION SCENARIOS AND IMPLEMENTATION}

\section{A. Real Time Reminders}

Generally, the context engine can be used in the following three ways: real time reminders, applications using the context engine, and daily life record.

TABLE I: EXAMPLES OF REMINDER

\begin{tabular}{ll}
\hline \hline Reminders & Explanation and example \\
\hline Falls & $\begin{array}{l}\text { Notification by phone call or SMS to the user's } \\
\text { guardians if the user has fallen down. }\end{array}$ \\
Exercise & $\begin{array}{l}\text { Remind the user to stand or walk if he/she has sat for } \\
\text { more than a specified period of time (e.g. } 2 \text { hours). }\end{array}$ \\
$\begin{array}{l}\text { Sleeping } \\
\text { Monitoring }\end{array}$ & $\begin{array}{l}\text { Sleeping without any movements for more than four } \\
\text { hours may indicate that the user has fallen into a } \\
\text { comma state. }\end{array}$ \\
$\begin{array}{l}\text { Environment } \\
\text { al Amenity }\end{array}$ & $\begin{array}{l}\text { Notification of the user whenever he/she enters an } \\
\text { environmental amenity that is suboptimal. }\end{array}$ \\
\hline \hline
\end{tabular}

The context engine can give real-time reminders or alarms to the user or to the user's guardian and doctors according to the environment, the user's activity, the user's physical condition according and the predefined rules set. Experiment show that these reminders can be very helpful to user so long as these rules are appropriately configured. These reminders cover scenarios such as the user's daily activity, environmental impact, abnormal vital signs, etc. The following are some examples of the reminders tested in the context engine (see Table I).

All the reminders work under predefined rules. Here is an example of predefined rule in the context engine reminding the user to avoid from being exposed to the sun when the ultraviolet ray intensity abstract value is greater than 1 .

If (ult001 > 1) Remind ("ultraviolet ray intensity is harmful, Avoid being exposed to the sun")

\section{B. Applications Based on the Context Engine}

These applications are not included in this context engine, they are potential applications developed by other developers and can run on android while using the data collected by the context engine. One of reason the context engine is needed is to solve the problem of conflict when two or more applications require for the same hardware sensor resource at the same time. The context engine can collect the sensed data once and provide for all the applications requiring data. In the incorporated design, these applications can request for data from the context engine in the following three ways. a) Send the data of information entity $x$ now. b) If the value of information entity $\mathrm{x}$ is below $\mathrm{y}$, provide notification. c) 
Record the value of information entity $\mathrm{x}$ for the next minutes and send the result data then. Therefore some APIs are provided in the context engine for these applications. The following are some examples:

final static informationEntityValue getInformationEntityValue (int threadId, String informationEntity, int abstractLevel, String description),

final static int reqeustNotIfication (int threadId, String informationEntity, int abstractLevel,String condition ),

final static void sendNotIfication (int threadId, String notIfication),

final static int reqeustDataPeriod (int threadId, String informationEntity, int abstractLevel, Date beginningTime, int timespan)

By using the data collected through context engine, these applications can provide more professional and specialized service by focusing on only a special kind of issue faced by the user. For instance, a user with diabetes and a user with senile dementia would need different services provided by the applications.

For instance, here are a sample instruction for diabetes patients:

Test blood glucose 3 times a week 2 hours before or after meal.

The following is a peace of pseudo code using the API. activity=getInformationEntityValue(Process.myTid(),'act01', Level2!,")

If $($ day $==$ Monday! $\|$ day $==$ Wednesday $\|$ day $==$ Friday! $)\{$ If $($ Activity $==$ Breakfast! $)\{$

Remind("today is your blood glucose taking day. Please take your blood glucose 2 hours after breakfast.")

time $=\operatorname{now}()$

\}

If (now () - time) $>=110$ minutes $)\{$

Remind("please take your blood glucose in around 10 minutes")

\}

\}

\section{Daily Life Logging}

The record of the user's vital signs and daily activity is very valuable for the user because human memory and feelings felt are not always reliable. For instance, it's not easy to give an accurate answer to the doctor when asked, "How many hours of exercise did you do in the last week?" Since the context engine actually collects this data about the user's daily life, it becomes possible to record all the useful data collected just as an operating system logs every activity in a system log. These records can provide as an evidence-based recourse for the future medical treatment

In the context engine, data of user's vital signs and daily activities are recorded because they are the most important data for the user's future medical treatment. Vital signs include user's blood pressure, body temperature, blood glucose and other necessary vital signs. Daily activity include activities such as going to bed, getting up, meal taking, walking, sitting, standing, sleeping, jogging, smoking, going to bathroom, etc. From this, professionals can calculate the user's daily routine and frequency of activities.

\section{CONCLUSION}

In this paper, a health care oriented context engine for the mobile platform is introduced. It collects data from three types of sensors. The context information is handled as information entity and abstracted to higher level data. The context engine provides real time reminders to user and share context information with other applications run on the same platform.

The context engine presented brought up the idea of collecting as much as possible health care related information that can be sensed, and organizing it with the portable smart phones to cover as much of user's daily life time. It can be then used to improve the life quality of senior citizens and help them to live more independently.

\section{ACKNOWLEDGMENT}

This research was supported by Basic Science Research Program through the National Research Foundation of Korea (NRF) funded by the Ministry of Science, ICT and future Planning (grant number).

\section{REFERENCES}

[1] L. Daniele, E. G. D. Silva, L. F. Pires, and M. V. Sinderen, "A soa-based platform-specific framework for context-aware mobile applications," IWEI, 2009, pp. 25-37.

[2] G. Virone, A. Wood, L. Selavo, Q. Cao, L. Fang, T. Doan, Z. He, R. Stoleru, S. Lin, and J. A. Stankovic, "An assisted living oriented information system based on a residential wireless sensor network," in Proc. IEEE 1st Conf. on Distributed Diagnosis and Home Healthcare (D2H2 '06), April 2006, pp. 95-100.

[3] B. Andò, S. Baglio, S. L. Malfa, A. Pistorio, and C. Trigona, "A smart wireless sensor network for AAL," Measurements and Networking Proceedings (M\&N), 2011 IEEE International Workshop.

[4] S. Chiriac, N. Roll, J. Parada, and B. Rosales, "Towards combining validation concepts for short and long-term ambient health monitoring," presented at 2012 6th International Conference on Pervasive Computing Technologies for Healthcare (Pervasive Health).

[5] F. Paganelli and D. Giuli, "An ontology-based context model for home health monitoring and alerting in chronic patient care networks," presented at AINAW '07. 21st International Conference on Advanced Information Networking and Applications Workshops, 2007.

[6] M. T. Segarra, "Building a context-aware ambient assisted living application using a self-adaptive distributed model," presented at Fifth International Conference on Autonomic and Autonomous Systems, 2009.

[7] B. V. Wissen, N. Palmer, R. Kemp, T. Kielmann, and H. Bal, "ContextDroid: an expression-based context framework for Android," in Proc. Phone Sense 2010, Nov. 2010.

[8] D. Kramer, A. Kocurova, and S. Oussena, "An extensible, self-contained, layered approach to context acquisition," presented at ACM, New York, NY, USA 2011.

[9] M. Zhang and A. A. Sawchuk, "Human daily activity recognition with sparse representation using wearable sensors," IEEE Journal of Biomedical and Health Informatics, vol. 17, issue 3, 2013

[10] J. Aryo and D. Lai, "A prototype ZigBee to Bluetooth gateway for emerging body area network application," presented at 20114 th Annual Caneus on Fly by Wireless Workshop (FBW).

[11] L. C. Liu, S. M. Kuo, and M. C. Zhou, "Virtual sensing techniques and their applications," presented at International Conference on Networking, Sensing and Control, 2009.

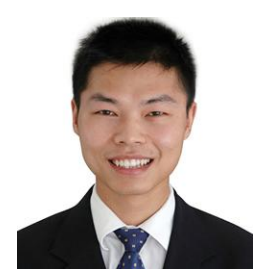

Zheng Guangbin received the BS degree in logistics engineering from Shandong university, China, in 2005. He had worked for Shanghai Wooriul Spine Hospital as the information department director from 2007 to 2012. He is currently pursuing the Master's degree in computer science department at Yonsei University, Seoul, Korea. His research interests include context engine, context aware application, human activity recognition in WSN and and ubiquitous computing. 


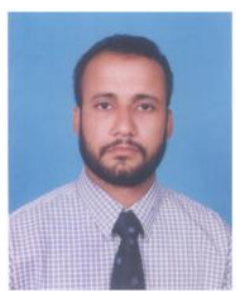

Muhammad Arshad Awan received the B.Sc. degree in mathematics and physics as major from University of the Punjab, Lahore, Pakistan and the M.Sc. and M.S. degrees in computer science from Quaid-i-Azam University, Islamabad, Pakistan and Allama Iqbal Open University, Islamabad, Pakistan respectively. He is currently pursuing the Ph.D. degree in computer science at Yonsei University, Seoul, Korea. His research interests include human activity recognition in WSN, semantic technologies, ontology enabled context management, and ubiquitous computing.

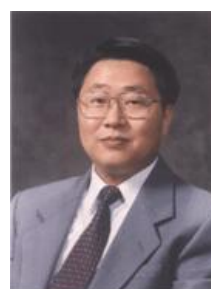

Shin-Dug Kim received the BS degree in electronic engineering from Yonsei University, Seoul, Korea, in 1982, and the MS degree in electrical engineering from University of Oklahoma in 1987. In 1991, he received the $\mathrm{PhD}$ degree from the School of Computer and Electrical Engineering at Purdue University, West Lafayette, Indiana. He is currently a professor of computer science at Yonsei University, Seoul, Korea.

His research interests include advanced computer architectures, intelligent cache/memory systems, embedded systems, and ubiquitous computing. 